\title{
Cj's Portable Model Pouring Vibrator: a Multipurpose Tool
}

\author{
Dr. Chiranjeev saini ${ }^{1}$, Dr. Elizabeth moirangthem ${ }^{2}$ \\ ${ }^{I}$ (Department of orthodontics, Rajasthan dental college and hospital,Jaipur,India) \\ ${ }_{2}^{2}$ (Department of pedodontics, Rajasthan dental college and hospital,Jaipur,India)
}

\begin{abstract}
Many innovations have enhanced the profession of orthodontics since the days of Edward. $H$. Angle. Diagnostics models are most important key tool in diagnosis \& treatment planning. Study models should be neat, clean and free from bubbles, so conventional vibrator is used while pouring. Here is a cost effective vibrator which can be made with simple items in just minutes, few bucks and it is cordless, rechargeable. It is just a body kneader; yet an innovation modified that can be used as a laboratory vibrator
\end{abstract}

Keyword: vibrator, portable, model pouring

\section{Introduction}

Diagnostic models are key tool for treatment planning, and good, neat, bubble free clean records are mandatory for postgraduate students. So the conventional vibrator for pouring is used. This is a portable pouring vibrator which is light weight, very cheap to fabricate and it is cordless. This vibrator is a body massager which is modified to a model pouring vibrator. This massager is easily available in market. It is reachable, battery operated and USB operated too. Continuous vibrations to pour an impression in not needed, so just 4-5 second vibration is sufficient so that the scoop of dental plaster flows down in to occlusal area and bubbles comes out ; this device is equipped with push action start up ,so it stops automatically after 4-5 seconds, allows you to pour next scoop of plaster on impression. Sufficient vibrations are delivered by the device.

1.USB power.

\section{Specifications}

2. Battery operated.

3. Chargeable

Key features -

1. Impression tray itself get lock on platform in tray holding notch, so no need to hold tray.

2. Push action; just push a little down it starts, so no on/off switch problem.

\section{Uses}

Uses: - Pouring ideal casts, pouring impression, vibrating material in bowl.

\section{STEPS IN CONSTRUCTION}

- Body Massager to be used as a main skeletal device, two used lids of jar \& feviquick Fig. 1.

- Base and top of the body massager and top of lids are scrapped and made rough for good adaptation and retention.

- Top of the lid is pasted to bottom and top of the body massager. Fig. 2.

- A notch exactly the size of impression try handle is cut of from the lid on the top. Fig. 3.

- Round Macintosh is cut exactly the inner size of the lid and placed, vibrator is ready to use . Fig. 4

\section{Working}

- Mixing of dental plaster in bowl and vibrating by just pushing down bowl with spatula. fig .5

- $\quad$ Pouring impression fig $.6 \&$ fig .7 


\section{Figures}

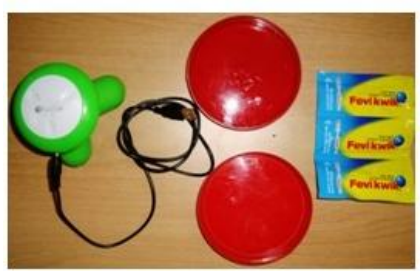

fig. 1

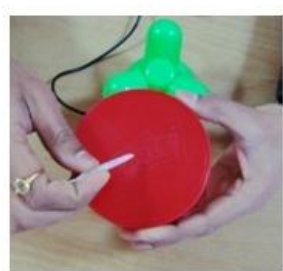

fig. 2

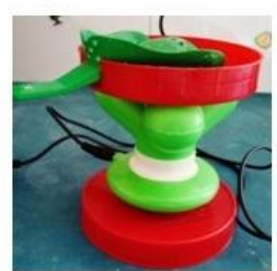

fig. 3

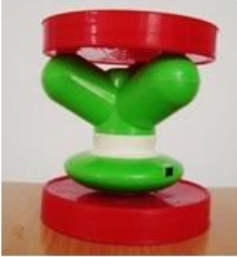

fig. 4

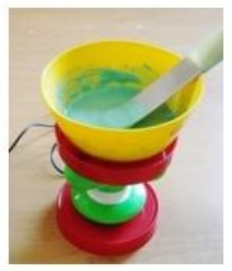

fig. 5

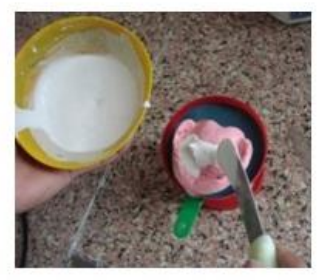

fig. 6

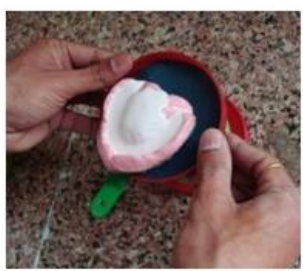

fig. 7

\section{Conclusion}

This model pouring vibrator is easy to fabricate, its portable, can be use anywhere, any time (without electricity) and most important cost almost nothing as compared to the conventional one. So this vibrator will start a new era of portable vibrating systems in dentistry. 